OPEN ACCESS

Edited by:

Akio Inui,

Kagoshima University Graduate

School of Medical and Dental

Sciences, Japan

Reviewed by:

Wayne B. Lau,

Thomas Jefferson University, USA Hiroshi Takeda,

Hokkaido University, Japan

Shunsuke Ohnishi,

Hokkaido University, Japan

*Correspondence:

Norio lizuka,

Department of Kampo Medicine,

Yamaguchi University Hospital,

1-1-1 Minami-Kogushi, Ube,

Yamaguchi 755-8505, Japan

iizuka@yamaguchi-u.ac.jp

Specialty section:

This article was submitted to Ethnopharmacology, a section of the journal

Frontiers in Pharmacology

Received: 07 September 2014

Accepted: 18 March 2015

Published: 08 April 2015

Citation:

lizuka $N$ and Hamamoto $Y(2015)$

Constipation and herbal medicine.

Front. Pharmacol. 6:73.

doi: 10.3389/fphar.2015.00073

\section{Constipation and herbal medicine}

\author{
Norio lizuka $^{1 *}$ and Yoshihiko Hamamoto ${ }^{2}$ \\ ${ }^{1}$ Department of Kampo Medicine, Yamaguchi University Hospital, Ube, Japan, ${ }^{2}$ Department of Computer Science \\ and Systems Engineering, Faculty of Engineering, Yamaguchi University, Ube, Japan
}

Constipation is characterized by a variety of bowel symptoms such as difficulty passing stool, hard stool, and a feeling of incomplete evacuation. The multifactorial causes of constipation limit the clinical efficacy of current conventional treatments that use a single drug that acts through only one pathway. To complement the shortcomings of the current Western medical model and provide a complete holistic approach, herbal medicines capable of targeting multiple organs and cellular sites may be used. In Japan, many herbs and herbal combinations have traditionally been used as foods and medicines. Currently, Japanese physicians use standardized herbal combinations that provide consistent and essential quality and quantity. This review highlights representative Japanese herbal medicines (JHMs), Rhei rhizoma-based JHMs including Daiokanzoto and Mashiningan, and Kenchuto-based JHMs including Keishikashakuyakuto and Daikenchuto, which coordinate the motility of the alimentary tract. This review provides a framework to better understand the clinical and pharmacological efficacies of JHMs on constipation according to the unique theory of Japanese traditional medicine, known as Kampo medicine.

Keywords: constipation, herb, herbal medicine, Kampo, traditional medicine

\section{Introduction}

Constipation is characterized by a variety of bowel symptoms such as difficulty passing stool, hard stool, and feelings of incomplete evacuation. It is reported that 12-19\% of Americans (Eoff, 2008) and $14 \%$ of Asians (Cheng et al., 2003) suffer from constipation, with symptoms varying by geographic location (Talley et al., 1993).

The multifactorial causes of constipation limit the clinical efficacy of current conventional Western treatments since these drugs act through a single pathway (Gallagher and O'Mahony, 2009). To complement these shortcomings and provide a complete holistic approach, herbal medicines capable of targeting multiple organ sites may be used (Suzuki et al., 2009). In Japan, many traditional herbs and herbal combinations are used as foods and medicines (Iizuka et al., 2003), and physicians currently use standardized herbal combinations with consistent quality and quantity of constituents (Yakubo et al., 2014).

This four-part review provides a framework to better understand the clinical and pharmacological efficacy of Japanese herbal medicines (JHMs) on constipation by: (1) explaining the unique theory of Japanese traditional medicine, or Kampo medicine (KM); (2) summarizing JHMs used for constipation; (3) explaining the clinical application and pharmacological action of Rhei Rhizoma-based JHMs; and (4) explaining the clinical application and pharmacology of Kenchuto-based JHMs.

\section{The Unique Theory of Japanese Traditional Medicine, or Kampo Medicine}

Kampo medicine is another term for traditional Japanese medicine based on traditional Chinese medicine (TCM; Tang et al., 2008). The history of KM can be traced to about the fifth or sixth 
TABLE 1 | Japanese herbal medicines (JHMs) and Kampo patterns.

\begin{tabular}{|c|c|c|c|c|c|c|c|c|}
\hline $\begin{array}{l}\text { Japanese herbal } \\
\text { medicines (JHMs) }\end{array}$ & Class & Kampo patterns & Qi deficiency & Qi stagnation & Qi counterflow & $\begin{array}{l}\text { Blood } \\
\text { deficiency }\end{array}$ & Blood stasis & Fluid excess \\
\hline Daiokanzoto & 1 & Intermediate & & & & & & \\
\hline Tokakujokito & 1 & Excess & & & & & $\bullet$ & \\
\hline Bofutsushosan & 1 & Excess & & $\bullet$ & & & & \\
\hline Tsudosan & 1 & Excess & & & & & $\bullet$ & \\
\hline Mashiningan & 1 & Intermediate & & & & & & \\
\hline Junchoto & 1 & Intermediate & & & & $\bullet$ & & \\
\hline Daikenchuto & 2 & Deficiency & $\bullet$ & & & & & \\
\hline Keishikasyakuyakuto & 2 & Deficiency & $\bullet$ & & & & & \\
\hline Shokenchuto & 2 & Deficiency & $\bullet$ & & & & & \\
\hline Keishikasyakuyakudaioto & 2 & Deficiency & $\bullet$ & & & & & \\
\hline
\end{tabular}

Class 1: Rhei-Rhizoma-based JHMs; Class 2: Kenchuto-based JHMs.

century, when TCM was likely introduced to Japan via Korea. At present, 148 government-regulated prescription JHMs are covered by Japanese national health insurance. These JHMs are highquality herbs in combinations and proportions fixed and standardized according to classical TCM literature (Watanabe et al., 2011). Concomitant use of JHMs and various Western drugs is permitted in daily clinical practice under Japanese national health insurance.

There is a marked difference between Western medicine and traditional Asian medicines such as TCM, KM, and traditional Korean medicine. Western medicine specifically and efficiently attacks abnormal organs or cells by targeting the cause of the disease. More specifically, Western medicine focuses on pathogenesis rather than host reaction. In contrast, KM is concerned with the host's reaction to the pathogen, and thus focuses on the host's inherent ability to promote health by targeting multiple organs or cells concurrently. This is largely due to the fact that JHMs contain a combination of herbs, and thus a vast array of constituents. An evidence-based approach fails to assess efficacy when studying individualized medicines under the KM system because the endpoints are somewhat unclear. This is an issue that requires further investigation in future studies.

Similar to TCM, KM uses patterns (Sho in Japanese, and Zheng in Chinese) to determine a suitable herbal combination for each patient. While TCM is based on the theory of the Ming Dynasty, $\mathrm{KM}$ was separated from this theory and then reestablished based on a different theory, Shang Han Lun, during the Edo period (Yakubo et al., 2014). While organ systems are very important for determining medication patterns in TCM, they are not utilized in KM because Japanese KM specialists wish to avoid overlap with terms used in Western medicine. Thus, KM patterns are quite unique. One possible reason for this may be the fact that TCM prescriptions are individualized at the herbal level, while $\mathrm{KM}$ prescriptions are individualized at the formula level (Yakubo et al., 2014).

For diagnosis in KM, several parameters are provided to formulate medication patterns, including yin-yang, deficiency-excess, cold-heat, interior-exterior, six stages of acute febrile diseases and Qi-blood-fluid (Sato et al., 2005; Yakubo et al., 2014). To assess and better understand patient status, KM defines chronic health conditions as, for example, deficiency, intermediate (i.e., between deficiency and excess), and excess patterns in the whole body and
Qi-blood-fluid. Qi, or life energy, is sourced from food and air. There are three types of abnormal Qi patterns: Qi deficiency, Qi stagnation, and Qi counterflow. Blood is a red fluid moved by Qi. There are two types of abnormal blood patterns: blood deficiency and blood stasis. Fluid, in contrast to blood, is a colorless and transparent liquid. In KM, illness is caused by an imbalance of these three elements. Under KM theory, constipation is thought to be caused mainly by a deficiency pattern, specifically Qi deficiency, and other patterns (intermediate or excess) caused by Qi stagnation, blood deficiency, or blood stasis (Table 1). Physicians specializing in $\mathrm{KM}$ can individualize constipation treatments using the following four traditional examination methods: inspection, listening and smelling, interviewing, and palpation (Sato et al., 2005).

\section{JHMs Used for Constipation}

Japanese herbal medicines are composed of various medicinal herbs. The pharmacological actions of JHMs in the body are largely determined by combinations and interactions between the herbs, which are used to treat KM patterns, including deficiencyexcess and Qi-blood-fluid. Figure 1 shows representative JHMs and their ingredients (i.e., herbs) used for constipation. There are two classes of JHMs, Rhei Rhizoma-based JHMs (class 1) and Kenchuto-based JHMs (class 2), both of which are frequently used for constipation (Figure 1; Table 1). Figure 2 also depicts the functional pharmacological relationship between Western drugs and the primary herbs used in JHMs. Information concerning herbs in KM were obtained from an online list of package inserts (http://plaza.umin.ac.jp/ kconsort/framepage.html).

Physicians specializing in KM usually use Rhei Rhizoma-based JHMs (class 1) to treat constipated patients with excess or intermediate patterns, most of whom show atonic constipation (Iizuka, 2011; Table 1; Figure 2). One possible reason for this is that sennoside A, aloe-emodin, and rhein, which are the main components of Rhei Rhizoma, have anti-inflammatory activities (He et al., 2013), and as a result, draw heat from the patient. Therefore, their pharmacological actions may cause harm in constipated patients with deficiency and cold patterns.

The word "Kenchuto" means an herbal combination that can improve the dysfunction of the alimentary tract (Sato et al., 2005). Kenchuto is classified into two types, Daikenchuto and 


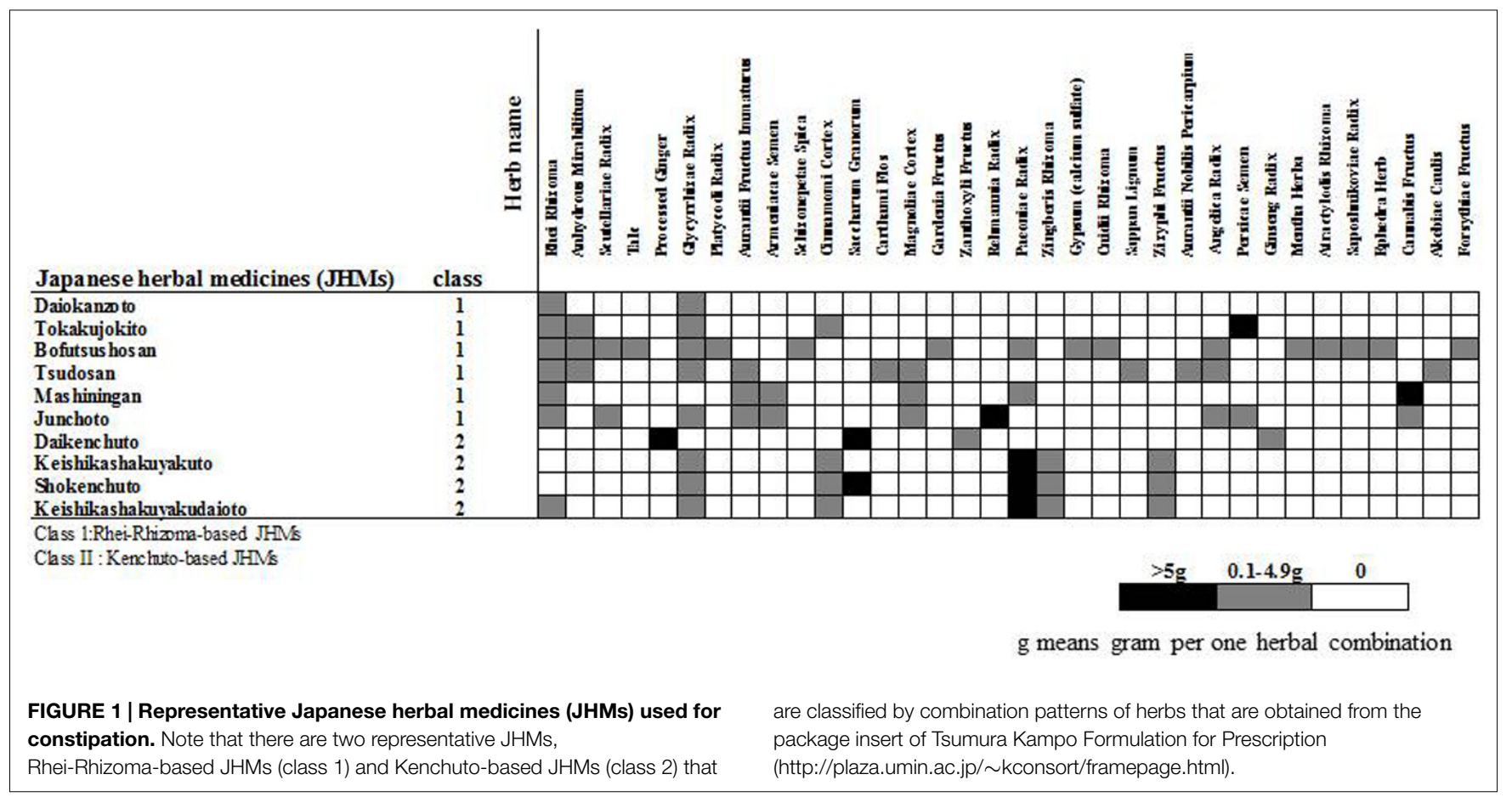

\section{Corresponding western drugs Main herb}

JHMs

(Japanese name)

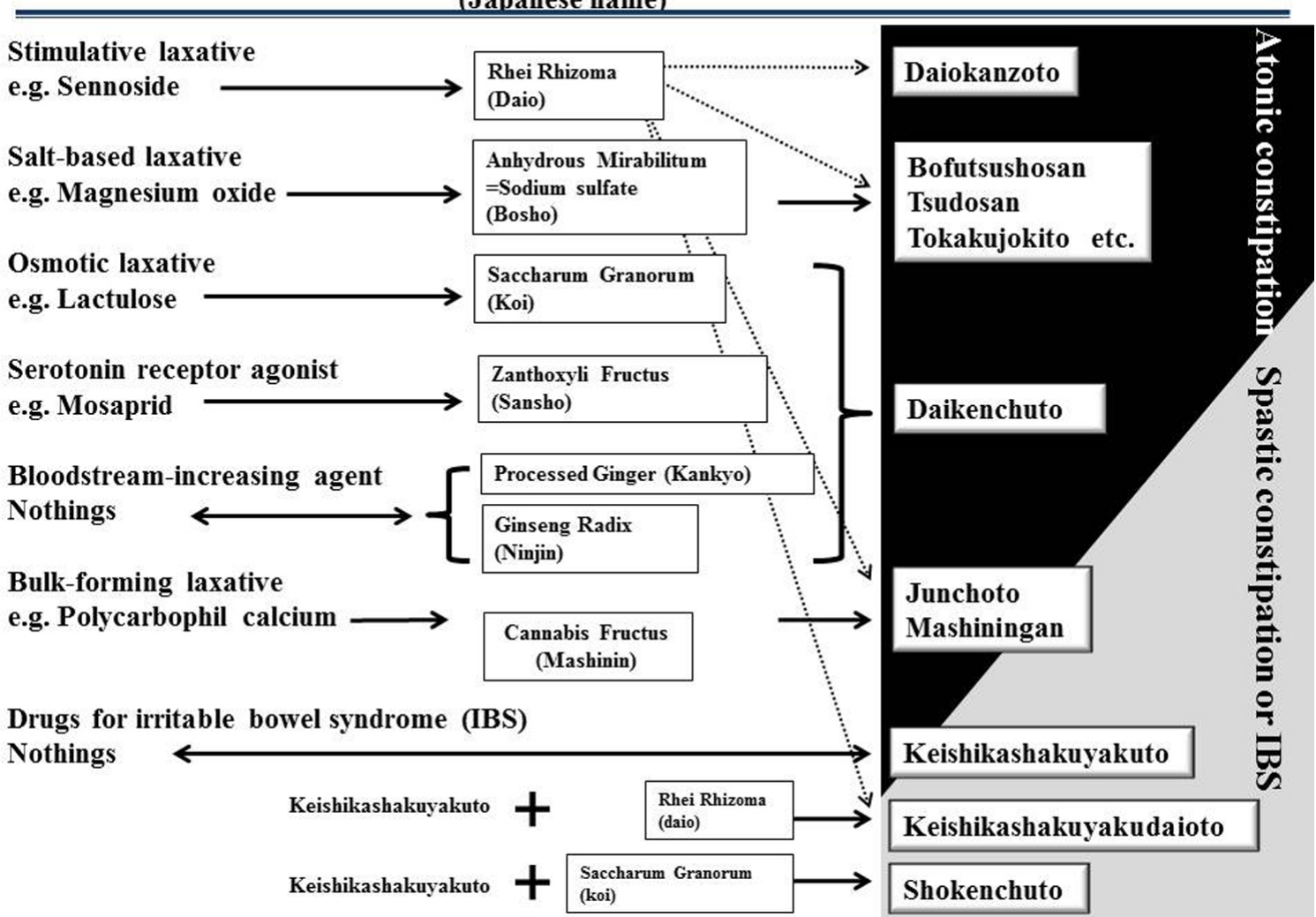

FIGURE 2 | Functional relation between Western drugs and Japanese herbal medicines (JHMs) used for constipation. JHMs and their main herbs are listed along with the corresponding Western drugs. Notably, none of laxatives increase bloodstream in the alimentary tract (adapted from lizuka, 2011). 
Keishikashakuyakuto, based on herb combinations. Interestingly, although these two types of JHMs have different herb combinations, they have similar functions in terms of promoting bowel movements (Figure 1). KM physicians typically use Kenchutobased JHMs (class 2) to treat constipated patients with deficiency and cold patterns who also report general fatigue due to Qi deficiency (Table 1). In Japan, Kenchuto-based JHMs are frequently prescribed when patients with deficiency and cold patterns develop constipation-predominant irritable bowel syndrome (IBS; Sato et al., 2005). In particular, contraction of the rectus abdominis muscle (Fukuhikokyu in Japanese) is often found in patients with constipation-predominant IBS. KM physicians recognize empirically that Paeoniae Radix, a main component of Kenchuto-based JHMs, promotes relaxation of the rectus abdominis muscle (Sato et al., 2005) as well as smooth muscle of the intestinal tract. Therefore, for patients with IBS, contractions of the rectus abdominis muscle can be used as a selection criterion for Kenchuto-based JHMs.

\section{Clinical Application and Pharmacology of Rhei-Rhizoma-Based JHMs}

Rhei Rhizoma (Rhubarb in English and Daio in Japanese) is one of the most frequently used herbs for constipation throughout the world. Rhei Rhizoma contains dianthrone glucosides (sennosides A to F) and anthraquinones (e.g., rhein, aloe-emodin, emodin, physcion, chrysophanol; Hardcastle and Wilkins, 1970; Dreessen et al., 1981; Ngoc et al., 2008). Among these components, sennosides (i.e., stimulative laxatives), have been well documented for their pharmacological action on constipation (de Witte, 1993). Sennosides A and B play a central role in the motility of the alimentary tract as prodrugs that are converted to an active principle, rheinanthrone, by intestinal microbiota (Hardcastle and Wilkins, 1970; Dreessen et al., 1981). A recent study showed that sennoside A may exert a laxative effect by inhibiting water transfer from the intestinal tract to the vascular side via decreasing aquaporin-3 expression in the colon (Kon et al., 2014).

Daiokanzoto is a representative Rhei rhizoma-based JHM that comprises two herbs (Rhei rhizoma and Glycyrrhizae radix). It is widely used to treat constipated patients with intermediate patterns (Table 1). The pharmacological action of Daiokanzoto appears similar to that of sennoside, a main component of Rhei Rhizoma (Figure 2). Inversely, a patient's response to sennoside may predict the clinical efficacy of Rhei Rhizoma-based JHMs. For example, KM physicians would not empirically prescribe Daiokanzoto for constipated patients deemed unresponsive to sennoside in the medical interview (Iizuka, 2011). Alternatively, Daiokanzoto has beneficial effects on oral mucositis, a disease that results from increased cell death induced by chemotherapeutic agent 5-fluorouracil (5-FU; Yoshida et al., 2014). Therefore, Daiokanzoto may improve both constipation and quality of life in cancer patients treated with 5-FU-based chemotherapy, although further studies are required to gain deeper insight into its pharmacological actions.

Bofutsushosan, Tsudosan, and Tokakujokito are composed of two main herbs: Rhei Rhizoma and Anhydrous Mirabilitum (Bosho in Japanese; Figures 1 and 2). Anhydrous Mirabilitum is a sodium sulfate similar to magnesium sulfate that acts as a saltbased laxative (Figure 2). Bofutsushosan, Tsudosan, and Tokakujokito are considered strong laxatives due to their stimulative and salt-based functions (Iizuka, 2011). Among these three JHMs, Tsudosan and Tokakujokito are used for constipated patients with blood stasis (Oketsu in Japanese; Table 1). Several herbs (Carthami Flos, Sappan Lignum, Angelica Radix, and Persicae Semen) used in Tsudosan and Tokakujokito improve blood stasis by inhibiting blood coagulation and causing vasodilation. For example, Angelica Radix contains coumarin derivatives, which have inhibitory effects on platelet aggregation and blood coagulation (Lee et al., 2003). Bofutsushosan is also used for constipated patients with Qi stagnation (Kitai in Japanese; Table 1). Daiokanzoto and these three JHMs are frequently used in patients with atonic constipation without deficiency and cold patterns.

Junchoto and Mashiningan are unique Rhei Rhizoma-based JHMs that contain small amounts of Rhei Rhizoma and Cannabis Fructus (Mashinin in Japanese; Figure 1). In Japan, KM physicians prescribe Junchoto and Mashiningan exclusively for elderly patients who have spastic constipation (Figure 2). Cannabis Fructus contains large amounts of fatty oils, including olein, linolein, and linolenin, with actions similar to the bulk-forming laxative, polycarbophil calcium (Montserrat-de la Paz et al., 2014; Figure 2). In most patients with constipation, Junchoto or Mashiningan soften stool. Generally, constipated stool is dry, hard, and difficult to pass. Therefore, the combination of Rhei Rhizoma, which promotes movement of the alimentary tract, and Cannabis Fructus, which softens stool, may prove effective for any type of constipation, including atonic and spastic constipation (Figure 2). Mashiningan has been proven efficacious for functional constipation in a randomized double-blind, placebocontrolled study (Cheng et al., 2011). In that study, 120 patients with functional constipation were randomized into two groups, a Mashiningan (Hemp Seed Pill) and a placebo group. Responder rates for the Mashiningan and placebo groups were 43.3 and $8.3 \%$ during treatment and 30.0 and $15.0 \%$ at 8 -week follow-up, respectively $(P<0.05)$. This suggests that Mashiningan increases complete spontaneous bowel movement, relieves the severity of constipation and straining to evacuate, and effectively reduces the need for a laxative.

\section{Clinical Application and Pharmacology of Kenchuto-Based JHMs}

Daikenchuto, one member of the Kenchuto-based JHM family, has a wide range of pharmacological actions and therefore is the most commonly prescribed JHM. It is composed of the following four herbs: processed ginger (Kankyo in Japanese), Ginseng Radix Rubra (Ninjin in Japanese), Zanthoxyli Fructus (Japanese pepper, Sansho in Japanese), and Saccharum Granorum (maltose powder derived from rice, Koi in Japanese; Kono et al., 2009; Figure 1). Due to a lack of relevant studies, the precise pharmacological action of Saccharum Granorum, a disaccharide with high osmotic pressure, remains unclear; however, it is not difficult to suspect that it might affect stool consistency and the motility of the alimentary tract in a manner similar to that of lactulose, an osmotic laxative (Figure 3). 


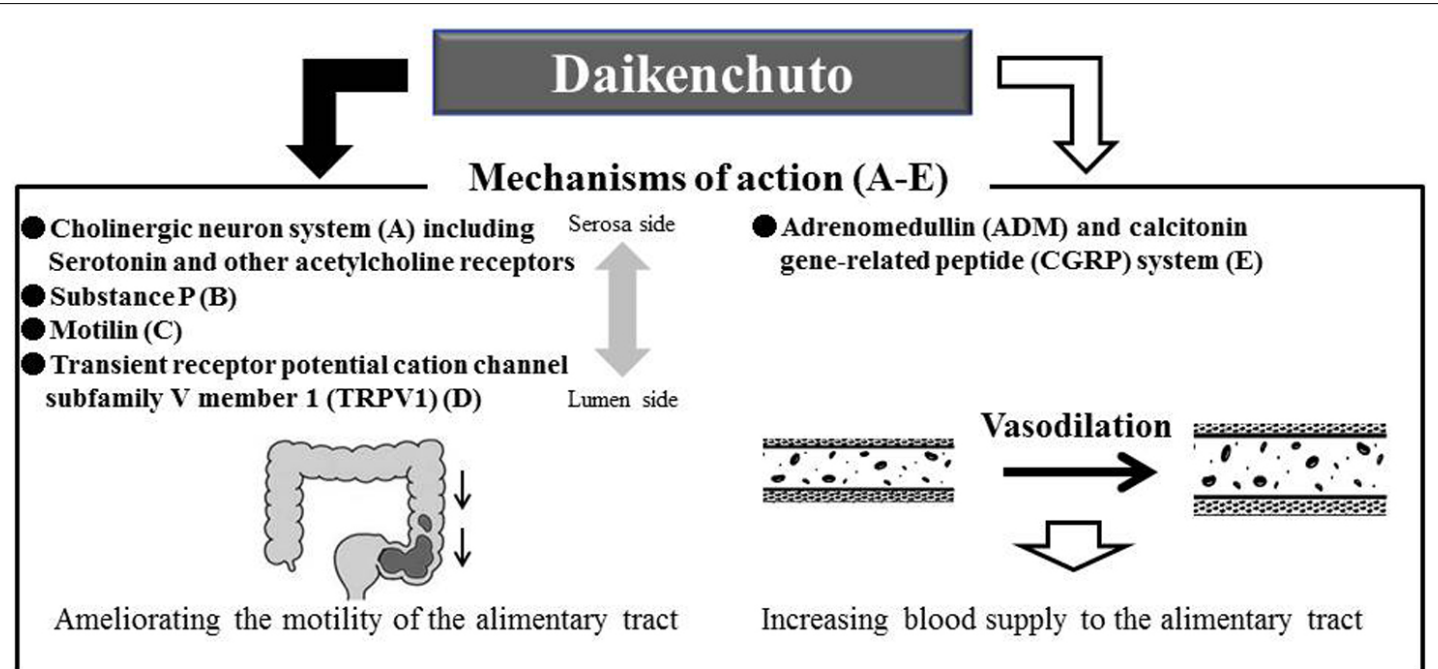

\begin{tabular}{|l|c|}
\hline & Corresponding mechanisms \\
\hline Daikenchuto (Whole drug) & $\mathbf{A} \sim \mathbf{E}$ \\
\hline Zanthoyli fructus and its component hydroxy-sanshool & $\mathbf{A , B , D , E}$ \\
\hline Processed ginger and its component shogaol & $\mathbf{D}, \mathrm{E}$ \\
\hline Ginseng radix and its component ginsenoside & $\mathbf{E}$ \\
\hline Saccharum granorum & - \\
\hline
\end{tabular}

FIGURE 3 | Two major mechanisms of action of Daikenchuto. Daikenchuto ameliorates the motility of the alimentary tract via four mechanisms (A to $\mathbf{D}$ ) and increases blood supply to the alimentary tract with use of adrenomedullin (ADM) and calcitonin gene-related peptide (CGRP) system (E). Notably, constituents playing an important role in individual mechanisms and system are identified.
The pharmacological actions of Daikenchuto, which are divided into two major putative mechanisms (Figure 3), have been well studied and documented (Shibata et al., 1999; Satoh et al., 2001a,b; Kono et al., 2008, 2009; Manabe et al., 2010). Daikenchuto increases blood supply to and improves the motility of the alimentary tract. The motility of the alimentary tract has been improved via the cholinergic neuron system (serotonin and nicotinic acetylcholine receptors), substance $\mathrm{P}$, motilin, and transient receptor potential cation channel, subfamily $\mathrm{V}$, member 1 (TRPV1) in several rodent and dog models with use of receptor antagonists or inhibitors (Nagano et al., 1999; Satoh et al., 2001a,b, 2003; Sato et al., 2004; Kikuchi et al., 2013). Several studies have shown that oral administration of Daikenchuto by healthy individuals increases plasma levels of substance P and motilin, which promote, either directly or indirectly, the motility of the alimentary tract (Nagano et al., 1999; Sato et al., 2004).

Zanthoxyli Fructus contains hydroxy-sanshools (alpha and beta), which act as a serotonin receptor agonist via the cholinergic neuron system to enhance intestinal peristalsis (Figure 3). As shown in Figure 2, its pharmacological action is similar to that of the serotonin receptor agonist mosapride. Indeed, it was shown that the increased intestinal motility induced by Daikenchuto was inhibited by several serotonin receptor antagonists (Shibata et al., 1999; Satoh et al., 2001a,b). A recent study by Kono et al. (2013) demonstrated that hydroxy-sanshools activate intestinal epithelial transient receptor potential cation channel, subfamily A, member 1 (TRPA1), which is highly expressed in enterochromaffin cells (serotonin-releasing cells), and may prove to be a novel target for constipation. In addition to the effects of these individual constituents, a recent study showed that Daikenchuto activates nicotinic acetylcholine receptors, which accounts for its effects on motility (Endo et al., 2014). Kikuchi et al. (2013) reported that pretreatment with atropine, hexamethonium, ondansetron (5-hydroxytryptamine-3 receptor antagonist), or capsazepine (TRPV1 antagonist), inhibited Daikenchuto-induced colonic contractions in a dog model. This suggests that orally administered Daikenchuto stimulates colonic motility via TRPV1, muscarinic, nicotinic, and 5-hydroxytryptamine-3 receptors. TRPV1, formerly known as vanilloid receptor 1 , is known as the capsaicin receptor. Interestingly, 6-gingerol and 6-shogaol, which are extracted from processed ginger, have vanilloid structures, and possibly act as TRPV1 stimulators (Figure 3). It has been reported that hydroxy-sanshools extracted from Zanthoxyli Fructus also bind to TRPV1 (Koo et al., 2007). These constituents play an important role in promoting the movement of alimentary tract via sensory nerves.

Unlike other JHMs used for constipation, Daikenchuto also increases blood circulation (Figure 3). To the best of our knowledge, no other pharmaceutical laxatives produce this effect. Increased intestinal blood supply is very fascinating from the standpoint of long-term maintenance of digestive function. As shown in Figures 2 and 3, both 6-shogaol extracted from processed ginger and hydroxyl-alpha-sanshool extracted from Zanthoxyli Fructus, have been shown to increase intestinal 
circulation via calcitonin gene-related peptide (CGRP) and adrenomedullin (ADM; Kono et al., 2008). Ginseng Radix has also been shown to increase blood supply to other organs, including brain tissues (Kim et al., 2002). These findings help explain why KM physicians frequently use Daikenchuto to treat constipated patients with deficiency and cold patterns from poor blood supply.

Both gingerols and shogaols, constituents of processed ginger, have anti-inflammatory and circulatory effects in the alimentary tract via modulation of mitogen-activated protein kinase (MAPK), protein kinase B (Akt), and NF- $\mathrm{B}$ activities (Hung et al., 2009; Wu et al., 2010; Huang et al., 2011; Li et al., 2013). Daikenchuto has been shown to significantly attenuate mucosal damage and adhesion in inflamed colon, and to inhibit elevated levels of proinflammatory cytokines TNF $\alpha$ and IFN $\gamma$ in the colon in animal models of Crohn's disease (Kono et al., 2010). This pharmacological action is explained in part by increased levels of ADM in small and large intestinal epithelial cells after Daikenchuto administration (Kono et al., 2010). These findings suggest that Daikenchuto has anti-colitis effects via the orchestration of several anti-inflammation pathways, which makes it suitable for treating patients with IBS or other inflammatory bowel diseases. The clinical application of these findings is currently underway in many hospitals throughout Japan, and the clinical efficacy of Daikenchuto will be elucidated in future studies.

Secondary members of the Kenchuto-based JHM family include Keishikashakuyakuto, Keishikashakuyakudaioto, and Shokenchuto, which are more frequently used in patients with constipation-predominant IBS (Sato et al., 2005; Oka et al., 2014). It is intriguing that Keishikashakuyakuto has shown antidiarrheal effects via the inhibition of excessively accelerated small intestinal movement (Saitoh et al., 1999); it has also been effective for relieving abdominal pain in patients with diarrhea-predominant IBS (Sasaki et al., 1998). Taken together, these reports suggest that Keishikashakuyakuto likely normalizes both accelerated and inhibited intestinal movements. This dual effect illustrates how certain herbal combinations, such as JHMs with multiple components, can help maintain host homeostasis.

Keishikashakuyakuto is composed of the following five herbs: Cinnamomi Cortex, Paeoniae Radix, Zingiberis Rhizoma, Zizyphi Fructus, and Glycyrrhizae Radix (Figure 1). Among these herbs, Paeoniae Radix and Glycyrrhizae Radix play a central role in ameliorating bowel dysfunction in patients with IBS. These two herbs has been shown to suppress the neurogenic contractions of the ileum induced by electrical stimulation and ganglionicstimulating agents in guinea pigs via inhibition of acetylcholine (Ach) release from cholinergic nerves and inhibition of Ach action on ileum smooth muscle (Maeda et al., 1983). Likewise, their antispasmodic effect on the human colon has been confirmed (Ai et al., 2006).

In addition to its spasmolytic and smooth muscle-relaxing effects (Takagi and Harada, 1969b; Hayashi et al., 1990; Tsuji et al., 2012), Paeoniae Radix has been shown to have anti-inflammatory and analgesic effects, to inhibit gastric acid secretion and stressinduced ulceration (Takagi and Harada, 1969a), and to have sedative (Takagi and Harada, 1969a,b) and antidepressant-like effects in rodent models (Mao et al., 2012). Mao et al. (2012) suggested that Paeoniae Radix has antidepressant-like effects, which could be mediated in part by inhibiting monoamine oxidase activity, hypothalamic-pituitary-adrenal axis activation, oxidative stress, and upregulated brain-derived neurotrophic expression.

Glycyrrhizin, a main component of Glycyrrhizae Radix, and its metabolite, $18 \beta$-glycyrrhetinic acid, have been reported as likely responsible for ameliorating dysfunctional glutamate transport in astrocytes via the inhibition of protein kinase activity (Kawakami et al., 2010). It has also been reported that after administration of $18 \beta$-glycyrrhetinic acid, about $13 \%$ passes through the bloodbrain barrier (Mao et al., 2012). These findings suggest that $18 \beta$ glycyrrhetinic acid in the brain can scavenge excess glutamate via a transporter, which might be related to the pathophysiology of bipolar disorder, major depressive disorder (Hashimoto et al., 2007), and schizophrenia (Schobel et al., 2013). Interestingly, brain-gut interactions have been suggested to play a central role in the pathogenesis of IBS (Fichna and Storr, 2012). Therefore, Keishikashakuyakuto orchestrates brain-gut interactions in IBS patients, Paeoniae Radix, in addition its direct effect on the alimentary tract, has both sedative and antidepressant-like effects, and Glycyrrhizae Radix induces upregulation of astrocyte glutamate transport. Thus, JHMs prove useful because they are capable of targeting multiple and/or distant organs such as the brain or alimentary tract concomitantly.

Shokenchuto is composed of the five herbs used in Keishikashakuyakuto and Saccharum Granorum (Figures 2 and 3). Keishikashakuyakudaioto is composed of the five herbs used in Keishikashakuyakuto, plus a small amount of Rhei Rhizoma (Figures 2 and 3). Considering that Saccharum Granorum and Rhei Rhizoma both enhance intestinal movement, it seems that Shokenchuto and Keishikashakuyakudaioto are stronger laxatives than Keishikashakuyakuto. However, the manner in which these three JHMs play important roles in controlling intestinal movement remains unclear. Further studies are needed to elucidate their specific pharmacological action on constipation.

\section{Conclusion}

The authors have reviewed classic JHMs used to treat constipation and compared the pharmacological actions of KM with those of Western drugs. In a single-center, randomized, and doubleblind study, Daikenchuto was found to have no significant effect on gastrointestinal and colonic transit, rectal compliance, anal sphincter pressure or bowel function in female patients with functional constipation (Iturrino et al., 2013). The effectiveness of KM allows physicians to provide individualized treatment to constipated patients and offer holistic JHMs capable of targeting multiple organ and cellular sites. However, there are limitations in the evaluation of the clinical efficacy of JHMs in a randomized clinical study setting because a placebo formulation that matches the texture, flavor, and other characteristics of the active drug is not always available. In addition, it is difficult to accurately evaluate herbal efficacy on individual patients with the current study model. A new strategy for evaluating various herbal combinations, including JHMs, and exploring pharmacological data may be needed in order to gain deeper insight into current herb-based therapies. 


\section{References}

Ai, M., Yamaguchi, T., Odaka, T., Mitsuhashi, K., Shishido, T., Yan, J., et al. (2006). Objective assessment of the antispasmodic effect of shakuyaku-kanzo-to (TJ-68), a Chinese herbal medicine, on the colonic wall by direct spraying during colonoscopy. World J. Gastroenterol. 12, 760-764. doi: 10.3748/wjg.v12.i5.760

Cheng, C., Chan, A. O., Hui, W. M., and Lam, S. K. (2003). Coping strategies, illness perception, anxiety and depression of patients with idiopathic constipation: a population-based study. Aliment. Pharmacol. Ther. 18, 319-326. doi: 10.1046/j.1365-2036.2003.01663.x

Cheng, C. W., Bian, Z. X., Zhu, L. X., Wu, J. C., and Sung, J. J. (2011). Efficacy of a Chinese herbal proprietary medicine (Hemp Seed Pill) for functional constipation. Am. J. Gastroenterol. 106, 120-129. doi: 10.1038/ajg.2010.305

de Witte, P. (1993). Metabolism and pharmacokinetics of anthranoids. Pharmacology 47, 86-97. doi: 10.1159/000139847

Dreessen, M., Eyssen, H., and Lemli, J. (1981). The metabolism of sennosides A and $\mathrm{B}$ by the intestinal microflora: in vitro and in vivo studies on the rat and the mouse. J. Pharm. Pharmacol. 33, 679-681. doi: 10.1111/j.2042-7158.1981. tb13903.x

Endo, M., Hori, M., Ozaki, H., Oikawa, T., and Hanawa, T. (2014). Daikenchuto, a traditional Japanese herbal medicine, ameliorates postoperative ileus by antiinflammatory action through nicotinic acetylcholine receptors. J. Gastroenterol. 49, 1026-1039. doi: 10.1007/s00535-013-0854-6

Eoff, J. C. (2008). Optimal treatment of chronic constipation in managed care: review and roundtable discussion. J. Manag. Care Pharm. 14, 1-15.

Fichna, J., and Storr, M. A. (2012). Brain-gut interactions in IBS. Front. Pharmacol. 3:127. doi: 10.3389/fphar.2012.00127

Gallagher, P., and O’Mahony, D. (2009). Constipation in old age. Best Pract. Res. Clin. Gastroenterol. 23, 875-887. doi: 10.1016/j.bpg.2009.09.001

Hardcastle, J. D., and Wilkins, J. L. (1970). The action of sennosides and related compounds on human colon and rectum. Gut 11, 1038-1042. doi: 10.1136/gut.11. 12.1038

Hashimoto, K., Sawa, A., and Iyo, M. (2007). Increased levels of glutamate in brains from patients with mood disorders. Biol. Psychiatry 62, 1310-1316. doi: 10.1016/j.biopsych.2007.03.017

Hayashi, M., Baba, K., and Maeda, T. (1990). Effect of shakuyaku-extract on electrical stimulation induced contraction of guinea pig ileum. Yakugaku Zasshi 110, 139-143.

He, X. Y., Liu, Q. C., Peng, W., Huang, Y. L., and Wu, C. J. (2013). Bioactivities and serum pharmacochemistry of Qi-Wei-Xiao-Yan-Tang. Pharm. Biol. 51, 629-634. doi: 10.3109/13880209.2012.761243

Huang, H. C., Chiu, S. H., and Chang, T. M. (2011). Inhibitory effect of [6]gingerol on melanogenesis in B16F10 melanoma cells and a possible mechanism of action. Biosci. Biotechnol. Biochem. 75, 1067-1072. doi: 10.1271/bbb. 100851

Hung, J. Y., Hsu, Y. L., Li, C. T., Ko, Y. C., Ni, W. C., Huang, M. S., et al. (2009). 6shogaol, an active constituent of dietary ginger, induces autophagy by inhibiting the AKT/mTOR pathway in human non-small lung cancer A549 cells. J. Agric. Food Chem. 57, 9809-9816. doi: 10.1021/jf902315e

Iizuka, N. (2011). Benpi. Daiokanzoto and Daikenchuto, Diagnosis and Treatment, Vol. 99. Tokyo: SHINDAN TO CHIRYO SHA Inc., 759-764 (in Japanese).

Iizuka, N., Oka, M., Yamamoto, K., Tangoku, A., Yamamoto, K., Miyamaoto, K., et al. (2003). Identification of common or distinct genes related to antitumor activities of a medicinal herb and its major component by oligonucleotide microarray. Int. J. Cancer 107, 666-672. doi: 10.1002/ijc.11452

Iturrino, J., Camilleri, M., Wong, B. S., Linker Nord, S. J., Burton, D., and Zinsmeister, A. R. (2013). Randomised clinical trial: the effects of daikenchuto, TU-100, on gastrointestinal and colonic transit, anorectal and bowel function in female patients with functional constipation. Aliment. Pharmacol. Ther. 37, 776-785. doi: 10.1111/apt.12264

Kawakami, Z., Ikarashi, Y., and Kase, Y. (2010). Glycyrrhizin and its metabolite $18 \beta$-glycyrrhetinic acid in glycyrrhiza, a constituent herb of yokukansan, ameliorate thiamine deficiency-induced dysfunction of glutamate transport in cultured rat cortical astrocytes. Eur. J. Pharmacol. 626, 154-158. doi: 10.1016/ j.ejphar.2009.09.046

Kikuchi, D., Shibata, C., Imoto, H., Naitoh, T., Miura, K., and Unno, M. (2013). Intragastric Dai-Kenchu-To, a Japanese herbal medicine, stimulates colonic motility via transient receptor potential cation channel subfamily V member 1 in dogs. Tohoku J. Exp. Med. 230, 197-204. doi: 10.1620/tjem.230.197
Kim, C. S., Park, J. B., Kim, K. J., Chang, S. J., Ryoo, S. W., and Jeon, B. H. (2002). Effect of Korea red ginseng on cerebral blood flow and superoxide production. Acta Pharmacol. Sin. 23, 1152-1156.

Kon, R., Ikarashi, N., Nagoya, C., Takayama, T., Kusunoki, Y., Ishii, M., et al. (2014). Rheinanthrone, a metabolite of sennoside A, triggers macrophage activation to decrease aquaporin-3 expression in the colon, causing the laxative effect of rhubarb extract. J. Ethnopharmacol. 152, 190-200. doi: 10.1016/j.jep.2013.12.055

Kono, T., Kaneko, A., Hira, Y., Suzuki, T., Chisato, N., Ohtake, N., et al. (2010). Anticolitis and -adhesion effects of daikenchuto via endogenous adrenomedullin enhancement in Crohn's disease mouse model. J. Crohns Colitis 4, 161-170. doi: 10.1016/j.crohns.2009.09.006

Kono, T., Kaneko, A., Omiya, Y., Ohbuchi, K., Ohno, N., and Ymamoto, M. (2013). Epithelial transient receptor potential ankyrin 1 (TRPA1) dependent adrenomedullin upregulates blood flow in rat small intestine. Am. J. Physiol. Gastrointest. Liver Physiol. 304, G428-G436. doi: 10.1152/ajpgi.00356.2012

Kono, T., Kanematsu, T., and Kitajima, M. (2009). Exodus of Kampo, traditional Japanese medicine, from complimentary and alternative medicines: is it time yet? Surgery 146, 837-840. doi: 10.1016/j.surg.2009.06.012

Kono, T., Koseki, T., Chiba, S., Ebisawa, Y., Chisato, N., Iwamoto, J., et al. (2008). Colonic vascular conductance increased by Daikenchuto via calcitonin generelated peptide and receptor-activity modifying protein 1. J. Surg. Res. 150, 78-84. doi: 10.1016/j.jss.2008.02.057

Koo, J. Y., Jang, Y., Cho, H., Lee, C. H., Jang, K. H., Chang, Y. H., et al. (2007). Hydroxy- $\alpha$-sanshool activates TRPV1 and TRPA1 in sensory neurons. Eur. J. Neurosci. 26, 1139-1147. doi: 10.1111/j.1460-9568.2007.05743.x

Lee, Y. Y., Lee, S., Jin, J. L., and Yun-Choi, H. S. (2003). Platelet anti-aggregatory effects of coumarins from the roots of Angelica genuflexa and A. gigas. Arch. Pharm. Res. 26, 723-726. doi: 10.1007/BF02976681

Li, X. H., McGrath, K. C., Tran, V. H., Li, Y. M., Duke, C. C., Heather, A. K., et al. (2013). Attenuation of proinflammatory responses by S-[6]-gingerol via inhibition of ROS/NF-Kappa B/COX-2 activation in HuH7 cells. Evid. Based Complement. Alternat. Med. 2013, 146142. doi: 10.1155/2013/146142

Maeda, T., Shinozuka, K., Baba, K., Hayashi, M., and Hayashi, E. (1983). Effect of shakuyaku-kanzoh-toh, a prescription composed of shakuyaku (Paeoniae Radix) and kanzoh (Glycyrrhizae Radix) on guinea pig ileum. J. Pharmacobiodyn. 6, 153-160. doi: 10.1248/bpb1978.6.153

Manabe, N., Camilleri, M., Rao, A., Wong, B. S., Burton, D., Busciglio, I., et al. (2010). Effect of daikenchuto (TU-100) on gastrointestinal and colonic transit in humans. Am. J. Physiol. Gastrointest. Liver Physiol. 298, G970-G975. doi: 10.1152/ajpgi.00043.2010

Mao, Q. Q., Ip, S. P., Xian, Y. F., Hu, Z., and Che, C. T. (2012). Anti-depressantlike effect of peony: a mini-review. Pharm. Biol. 50, 72-77. doi: 10.3109/ 13880209.2011.602696

Montserrat-de la Paz, S., Marín-Aguilar, F., García-Giménez, M. D., and FernándezArche, M. A. (2014). Hemp (Cannabis sativa L.) seed oil: analytical and phytochemical characterization of the unsaponifiable fraction. J. Agric. Food Chem. 62, 1105-1110. doi: 10.1021/jf404278q

Nagano, T., Itoh, H., and Takeyama, M. (1999). Effect of Dai-kenchu-to on levels of 3 brain-gut peptides (motilin, gastrin and somatostatin) in human plasma. Biol. Pharm. Bull. 22, 1131-1133. doi: 10.1248/bpb.22.1131

Ngoc, T. M., Minh, P. T., Hung, T. M., Thuong, P. T., Lee, I., Min, B. S., et al. (2008). Lipoxygenase inhibitory constituents from rhubarb. Arch. Pharm. Res. 31, 598-605. doi: 10.1007/s12272-001-1199-0

Oka, T., Okumi, H., Nishida, S., Ito, T., Morikiyo, S., Kimura, Y., et al. (2014). Effects of Kampo on functional gastrointestinal disorders. Biopsychosoc. Med. 8, 5. doi: 10.1186/1751-0759-8-5

Saitoh, K., Kase, Y., Ishige, A., Komatsu, Y., Sasaki, H., and Shibahara, N. (1999). Effects of Keishi-ka-shakuyaku-to (Gui-Zhi-Jia-Shao-Yao-Tang) on diarrhea and small intestinal movement. Biol. Pharm. Bull. 22, 87-89. doi: 10.1248/ bpb. 22.87

Sasaki, D., Uehara, A., Hiwatashi, N., Sekiguchi, T., Nakahara, A., Nakai, Y., et al. (1998). Kabinsei Cho Shoukougun ni taisuru, keishikashakuyakuto no rinsho kouka-tashisetsu kyodo musakui waritsuke gunkan hikaku rinsho shiken-(witten in Japanese). Jpn. J. Clin. Exp. Med. 75, 1136-1152.

Sato, Y., Hanawa, T., Arai, M., Cyong, J., Fukuzawa, M., Mitani, K., et al. (2005). Introduction to Kampo-Japanese Traditional Medicine. Tokyo: Elsevier Japan.

Sato, Y., Katagiri, F., Inoue, S., Itoh, H., and Takeyama, M. (2004). Dai-kenchu-to raises levels of calcitonin gene-related peptide and substance $P$ in human plasma. Biol. Pharm. Bull. 27, 1875-1877. doi: 10.1248/bpb.27.1875 
Satoh, K., Hashimoto, K., Hayakawa, T., Ishige, A., Kaneko, M., Ogihara, S., et al. (2001a). Mechanism of atropine-resistant contraction induced by Dai-kenchuto in guinea pig ileum. Jpn. J. Pharmacol. 86, 32-37. doi: 10.1254/jjp.86.32

Satoh, K., Hayakawa, T., Kase, Y., Ishige, A., Sasaki, H., Nishikawa, S., et al. (2001b). Mechanisms for contractile effect of Dai-kenchu-to in isolated guinea pig ileum. Dig. Dis. Sci. 46, 250-256. doi: 10.1023/A:1005636412287

Satoh, K., Kase, Y., Yuzurihara, M., Mizoguchi, K., Kurauchi, K., and Ishige, A. (2003). Effect of Dai-kenchu-to (Da-Jian-Zhong-Tang) on the delayed intestinal propulsion induced by chlorpromazine in mice. J. Ethnopharmacol. 86, 37-44. doi: 10.1016/S0378-8741(03)00038-2

Schobel, S. A., Chaudhury, N. H., Khan, U. A., Paniagua, B., Styner, M. A., Asllani, I., et al. (2013). Imaging patients with psychosis and a mouse model establishes a spreading pattern of hippocampal dysfunction and implicates glutamate as a driver. Neuron 78, 81-93. doi: 10.1016/j.neuron.2013.02.011

Shibata, C., Sasaki, I., Naito, H., Ueno, T., and Matsuno, S. (1999). The herbal medicine Dai-Kenchu-Tou stimulates upper gut motility through cholinergic and 5-hydroxytryptamine 3 receptors in conscious dogs. Surgery 126, 918-924. doi: 10.1016/S0039-6060(99)70033-4

Suzuki, H., Inadomi, J. M., and Hibi, T. (2009). Japanese herbal medicine in functional gastrointestinal disorders. Neurogastroenterol. Motil. 21, 688-696. doi: 10.1111/j.1365-2982.2009.01290.x

Takagi, K., and Harada, M. (1969a). Pharmacological studies on herb paeony root II. Anti-inflammatory effect, inhibitory effect on gastric juice secretion, preventive effect on stress ulcer, antidiuretic effect of paeoniflorin and combined effects with licorice component FM100. Yakugaku Zasshi 89, 887892.

Takagi, K., and Harada, M. (1969b). Pharmacological studies on herb paeony root III. Effects of paeoniflorin on circulatory and respiratory systems and isolated organs. Yakugaku Zasshi 89, 893-898.

Talley, N. J., Weaver, A. L., Zinsmeister, A. R., and Melton, L. J. III. (1993). Functional constipation and outlet delay: a population-based study. Gastroenterology 105, 781-790.
Tang, J. L., Liu, B. Y., and Ma, K. W. (2008). Traditional Chinese medicine. Lancet 372, 1938-1940. doi: 10.1016/S0140-6736(08)61354-9

Tsuji, S., Yasuda, K., Sumi, G., Cho, H., Tsuzuki, T., Okada, H., et al. (2012). Shakuyaku-kanzo-to inhibits smooth muscle contractions of human pregnant uterine tissue in vitro. J. Obstet. Gynaecol. Res. 38, 1004-1010. doi: 10.1111/ j.1447-0756.2011.01827.x

Watanabe, K., Matsuura, K., Gao, P., Hottenbacher, L., Tokunaga, H., Nishimura, K., et al. (2011). Traditional Japanese Kampo medicine: clinical research between modernity and traditional medicine-the state of research and methodological suggestions for the future. Evid. Based Complement. Alternat. Med. 2011, 513842. doi: 10.1093/ecam/neq067

Wu, H., Hsieh, M. C., Lo, C. Y., Liu, C. B., Sang, S., Ho, C. T., et al. (2010). 6-Shogaol is more effective than 6-gingerol and curumin in inhibiting 12-Otetradecanoylphorbol 13-acetate-induced tumor promotion in mice. Mol. Nutr. Food Res. 54, 1296-1306. doi: 10.1002/mnfr.200900409

Yakubo, S., Ito, M., Ueda, Y., Okamoto, H., Kimura, Y., Amano, Y., et al. (2014). Pattern classification in Kampo medicine. Evid. Based Complement. Alternat. Med. 2014, 535146. doi: 10.1155/2014/535146

Yoshida, K., Yoshioka, M., Okamura, H., Moriyama, S., Kawazoe, K., Grenier, D., et al. (2014). Preventive effect of Daiokanzoto (TJ-84) on 5-fluorouracil-induced human gingival cell death through the inhibition of reactive oxygen species production. PLoS ONE 9:e112689. doi: 10.1371/journal.pone.0112689

Conflict of Interest Statement: The authors declare that the research is partially supported by grants from TSUMURA \& CO.

Copyright (c) 2015 Iizuka and Hamamoto. This is an open-access article distributed under the terms of the Creative Commons Attribution License (CC BY). The use, distribution or reproduction in other forums is permitted, provided the original author(s) or licensor are credited and that the original publication in this journal is cited, in accordance with accepted academic practice. No use, distribution or reproduction is permitted which does not comply with these terms. 